\title{
Magnetic field evolves to gravity field part:4 Atomic structure
}

\author{
John C. Hodge ${ }^{1 *}$ \\ ${ }^{1}$ Retired, 477 Mincey Rd., Franklin, NC, 28734
}

October 11, 2019

\begin{abstract}
The Scalar Theory of Everything (STOE) suggests gravity emerges from magnetic effects of hods in matter structures. The suggested structure of electrons formed from disc magnets is expanded to describe atomic structure. The model improvement is the STOE atomic structure is more closely related to observations of Maxwell's Equations, of interference phenomena including those that reject wave models, and of cosmology through common basic components of the universe. The formation of atomic structure by magnetic rather than electric forces suggests all matter is composed of magnetic structures.
\end{abstract}

keywords: magnet, gravity, atomic structure, atomic spectra

\section{INTRODUCTION}

Atomic structure has been suggested by observations of atomic spectra (Condon and Odabasi 1980; Herzberg 1944). The emitted and absorption of spectra appear as lines in a spectrograph. The lines represent different energies of light. The lines appear as grouping of lines that are labeled "series". Some series, particularly lower energy series, overlap other series. Balmer found the relationship between lines of the series that bears his name could be calculated by:

$$
v=Z^{2} R\left(\frac{1}{n^{2}}-\frac{1}{n_{1}^{2}}\right),
$$

where $v$ is the wave number of the light which is proportional to energy, $R$ is the Rydberg constant which is different for each element, $Z$ is the atomic number, $n$ is an integer that denotes the series (called the "principle quantum number"), and $n_{1}$ is the line in the series. A line is the difference between $n$ and $n_{1}$. This

*E-mail: jchodge@frontier.com 


\section{INTRODUCTION}

works well for hydrogen and poorly for heavier elements. This concept uses arbitrarily assigned numbers to correspond to empirical data.

Bohr attempted to relate the $n$ of an atom and its structure. The RutherfordBohr model posits the atom consists of a heavy nucleus with a positive charge of $Z e$ that electrons revolve around at discrete classical orbits where $Z$ is the atomic number and $e$ the electric charge. Radiation is absorbed or emitted by the transition of electrons from one orbit to another. The light energy $E$ is $h \nu$ where $h$ is Planck's constant and $\nu$ is the light frequency in a wave model of light. Franck and Hertz observed experimentally in inelastic collisions the transfer of energy is equal to excitation energy of the spectrum.

An advantage of Bohr's concept is that the light emitted has an energy which is related to spectrographic data. A mass orbiting a center also has a calculable mass that is related to the radius of the orbit. The calculated radius for atomic electron spectra is of the same order of magnitude as the calculated "cloud" of electrons from collision experiments.

Because only certain orbits occur, an added postulate without justification was that the angular momentum is an integral multiple $n$ of $h / 2 \pi$. Thus, the radii $r$ of the possible orbits are proportional to $n^{2}$. The radius of the smallest orbit $(0.529 \AA)$ is the same order of magnitude of the radius of the atom. Several refinements include the idea the orbits can be elliptical that results in postulating a "azimuthal quantum number" $k$. Relativistic considerations resulted in a dependence of the energy on the "fine structure constant" $\left(\alpha=2 \pi e^{2} / h c\right)$

The $n$ and $k$ levels and sublevels should combine with other sublevels to correspond to observed energy difference or spectral lines. However, the number of observed components is much smaller than the expected number of the combinations. This discrepancy is treated by assumed so-called "selection rules". Even then there are deviations from the selection rules that suggested several new assumptions that are the addition of quantum numbers to $n$ and $k$.

The idea of electron orbiting the nucleus is contradicted by the experiments resulting in Maxwell's Equations. Accordingly, de Broglie suggested the electrons are waves and the circular or elliptical circumference of an "orbit" defined by its radius is the integer number of waves of the electron. Modern models of atomic spectra modify the Bohr/de Broglie model by adding additional quantum integer parameters to describe the variation in energy levels of lines. Empirical data required additional selection rules be assumed.

The emitted light is the difference in the number of waves. However, this model then presupposes the light has a wave nature. Although the wave nature of light is generally accepted, several experiments have rejected wave-like light in favor of particle (photon) light (Hodge project).

The Scalar Theory of Everything (STOE) posits the universe is composed of hods and plenum which emerge to form all in the universe (Hodge 2016b). The hods are discrete and the plenum is continuous (Hodge 2018e, figure 3.1). That is, discrete phenomena emerge from hod behavior and structure. Continuous phenomena emerge from plenum behavior and structure.

Charge models of electrons would have negative charges forming the electron. This structure would explode and forming such an electron structure 


\section{THE MODEL}

would require energy input. A magnetic structure would be a minimum of potential energy for the hods. It is, therefore, a stable structure conforming to the emergence of complex structures from simpler components.

Electric effects are vortices formed in the plenum by the movement of hods and hod structures through the plenum (Hodge 2018a). The STOE suggests the self-similarity principle that posits small size scales are similar to our everyday size scale. Accordingly, disk magnets were used to model photons and leptons (Hodge 2016a). According to the STOE, atomic spectra must be an emerged property of the hod structure. Therefore, atomic structure results from magnetic effects rather than electric effects.

The STOE rejected the Biot-Savart Law and suggested two types of magnetic effects (Hodge 2018b,c,d). One type emanates from magnets, the other from electric currents. The suggested model is that electrons (see Hodge (2016a)) have hairs (see Fig. 1) that shed hods as the electron travel through a wire which is one type of magnetic field.

The STOE model of a photon is a column of hods (Hodge project). This model and its simulation program successfully reproduced the interference experiments including those that rejected wave models of light. The spectrographic behavior of STOE photons is developed from the energy of photons being proportional to the number $N$ of hods in the column of hods. Each hod is a permanent magnet modeled by disc magnets. The photoelectric experiments relate the energy of photons proportional the wave model frequency of light. The slope of the resulting line is, in STOE terms, caused by the addition of one more hod to the photon. Therefore, the energy $E$ of a photon is $h^{\prime} N$ where $h^{\prime}=K h$ and $K$ is a proportionality constant between light frequency and $N$ determined spectrographically [sinc() function]. The model suggests the hods in the photon are a constant distance between them that is dependent of the average density of the surrounding plenum (Hodge 2012). That is, the photons have a length $l \propto N$.

This Paper suggests a STOE compatible model of atomic structure of disk magnets to represent hods to begin the enquiry. The description of the basic model is in section 2. The Discussion and Conclusion are in section 3 .

\section{THE MODEL}

If the spectrum of light is different for different light, the photons are of different length. If the light originates from emission from atoms, then different length photons are a part of the atomic structure. They are like rods holding the electrons and protons together by magnetism. The electric vortex field is caused by particle motion as the electrons and protons of an atom move. The magnetic field is stronger than the vortex field.

The connecting rod concept solves the lack of radiation of orbiting electrons without using waves of de Broglie or quantum numbers that have to be assumed ad hoc. The rods of hods then become directly linked to many other observations such as spectra and interference experiments. 


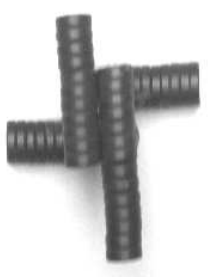

Figure 1: Picture of an electron with hairs.

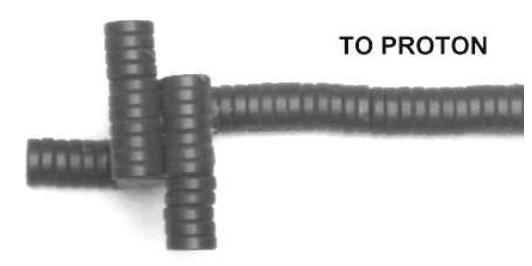

Figure 2: Picture of a hydrogen electron.

Figure 2 shows a model of the hydrogen electron bound to the nuclear proton. The electron is attached and becomes part of the magnetic structure of the electron. The other hairs are directions of possible attachment of other electrons or protons.

The rods are bar magnets with a stronger North Pole than the South Pole (see Table 1 of Hodge $(2019 \mathrm{~b})$ ). So, the rod connection of an electron to a proton (see Fig. 2) is slightly stronger if the North pole is the connection to the electron. This is the observed "spin" energy level.

Figure 3 shows a model of the helium atom atomic structure. The noted angle forms naturally in the magnetic structure of the electron. Because the nucleus has a small diameter relative to the length of the connecting photons, the angle has the outward directed photons diverging such that no other nucleus of 2 or more protons can connect. The connection of single protons $(2$ hydrogen atoms) may connect. However, the distance between them is sufficient that the electric vortex field generated by their movement would repel them away.

If the rods of photons are longer, then the angles are reduced so that another atom may also connect with rods to the same two or more electrons. This is the covalent bond as modeled in Fig. 4. Covalence bonding is formed by electrons in the second valence shell (of eight) or higher.

The photons in the top of the electron may form an angle between them (see Fig. 5). Figure 17 of Hodge (2019a) shows the magnetic field strength around the electron structure. The peaks occur every $45^{\circ}$. This field from the body helps twist the hairs. By forming the electron from disc magnets, without outside forces the angle $a$ formed is $20^{\circ} \pm 20^{\circ}$ if the internal height (see the brackets in Fig. 6) is seven discs high and $40^{\circ} \pm 10^{\circ}$ if the internal height is six discs high. That is, $0^{\circ} \leq a \leq 50^{\circ}$. The circle of electrons formed by an $a=45^{\circ}$ is eight electrons and by an $a=20^{\circ}$ is eighteen electrons - the "full" numbers 


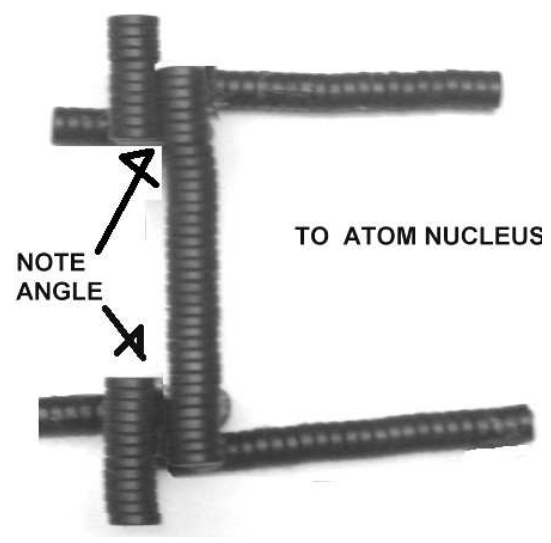

Figure 3: Picture of a helium electron pair.

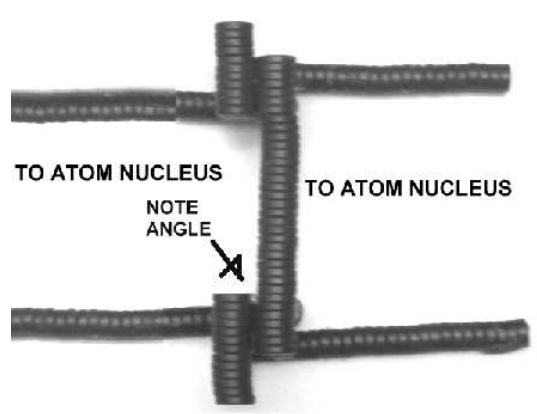

Figure 4: Picture of a covalent bond.

of shells 2,3 , and 4 .

If the number of electrons in the 2 shell is less than 8 , then a "hole" may exist such as seen in Fig. 5. An electron from another atom with a single valence electron may fill this hole by bonding with the electron hairs on either side rather than with photons in the nucleus.

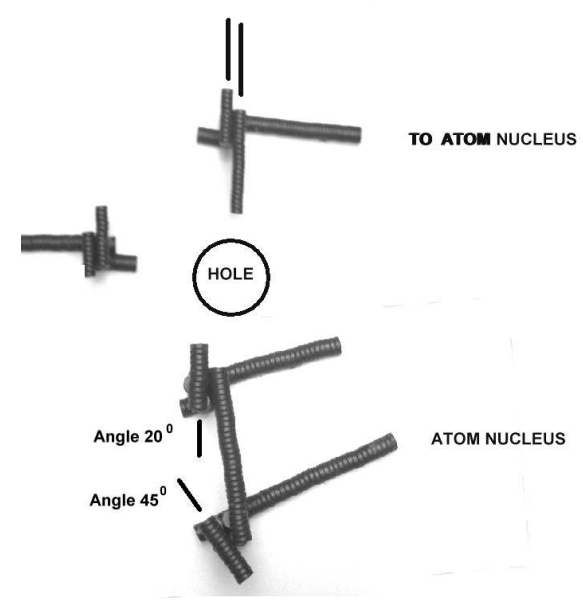

Figure 5: Picture of the electrons nearing an ionic bonding. 


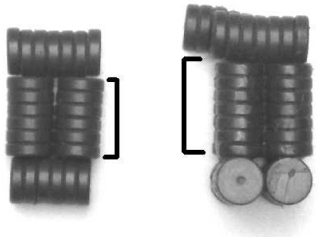

Figure 6: Picture showing the difference in the internal number of disks (hods) effect on height of the electron.

\section{DISCUSSION AND CONCLUSION}

The STOE proposes over several papers that phenomena of the small may be better explained by cause-and-effect analogy to our everyday world rather than by mysterious, weird statistics.

The suggested structure allows the calculation of the cord length $c$ between electrons in a shell by $c=2 l \sin (a / 2)$. This is the same kind of relation that de Broglie suggested that also avoids the violation of Maxwell's Equations. However, instead of invoking ellipsoid lengths, the STOE allows $a$ to change dependent on the number of electrons in a near circular orbit including ionic bound electrons and electrons from other atoms in a molecule. The $l$ and, hence, the $c$ may change depending on the $Z$ of the nucleus. The STOE suggests the $n$ terms of Eq. 1 are related through a circular structure of electrons, thus the $n^{2}$ and $Z^{2}$ as in the Bohr model.

The magnetic forces of the electron shells force a geometric structure on the electrons. The photon connection to the nuclear protons suggests this same structure of the protons. This leaves the remaining protons to form neutrons by a low energy binding of electrons and the other protons.

The cause of the number of hods in an emitted photon remains to be empirically determined. The model improvement is the STOE atomic structure is more closely related to observations of Maxwell's Equations, of interference phenomena including those that reject wave models, and of cosmology through common basic components of the universe. The formation of atomic structure by magnetic rather than electric forces suggests all matter is composed of magnetic structures.

ORCID iD

John C. Hodge: https://orcid.org/0000-0002-1520-2153 


\section{REFERENCES}

\section{References}

Condon, E.U., and Odabasi, H, 1980, Atomic Structure, (ISBN 052121859 4, (Cambridge University Press, New York, New York, USA).

Hertzberg, G., 1944, atomic spectra and atomic structure, (ISBN 13: 978-9-48660115-1, (Dover Publications, Inc., New York, New York, USA).

Hodge, J.C., 2012, Photon diffraction and interference, IntellectualArchive, Vol.1, No. 3, P. 20,. http://intellectualarchive.com/?link=item\&id=597

Hodge, J.C., 2016a, Structure and spin of the neutrino, electron, and positron, IntellectualArchive, Vol.5, No. 5, P. 1 ,. http://intellectualarchive.com/?link=item\&id=1694

Hodge, J.C., 2016b, STOE emergence, http://intellectualarchive.com/?link=item\&id $=1757$

Hodge, J.C., 2017, STOE simulation of photon spectrographic behavior, IntellectualArchive, Vol.6, No. 6, P. 1, http://intellectualarchive.com/?link=item\&id $=1884$

Hodge, J.C., 2018a, STOE electric charge, IntellectualArchive, Vol.7, No. 2, P 1. 1 , http://intellectualarchive.com/?link=item\&id=1917

Hodge, J.C., 2018b, Magnetostatics relation to gravity with experiment that rejects Biot-Savart Law, IntellectualArchive, Vol.7, No. 3, P. 1,. http://intellectualarchive.com/?link=item\&id=1945

Hodge, J.C., 2018c, Another experiment rejects Ampere's Law and supports the STOE model, IntellectualArchive, Vol.7, No. 4, P. 6,. http://intellectualarchive.com/?link=item\&id $=1956$

Hodge, J.C., 2018d, Two different types of magnetic field, IntellectualArchive, Vol.4, No. 4, P. 1,. http://intellectualarchive.com/?link=item\&id=1964

Hodge, J.C., 2018e, STOE replaces relativity and quantum mechanics, (ISBN 978-613-9-91465-4, (LAP LAMBERT Acedemic publishing, Mauritius, available through Amazon.com).

Hodge, J.C., 2019a, Magnetic field evolves to gravity field part:2 Particles, IntellectualArchive, Vol.8, No. 3, P. 30,. http://intellectualarchive.com/?link $=$ item\&id $=2170$

Hodge, J.C., 2019b, Magnetic field evolves to gravity field part:3 Electromagnets , IntellectualArchive, Vol.8, No. 4, P. 1,. http://intellectualarchive.com/?link=item\&id $=2193$

Hodge, J.C., Project, STOE photon diffraction and interference , https://www.researchgate.net/project/STOE-photon-diffraction-andinterference . 